\title{
Competitive 0 and $\pi$ states in S/F/S trilayers: Multimode approach
}

\author{
T. Karabassov, ${ }^{1}$ V. S. Stolyarov, ${ }^{2,3}$ A. A. Golubov, ${ }^{2,4}$ V. M. Silkin, ${ }^{5,6,7}$ V. M. Bayazitov, ${ }^{8}$ B. G. Lvov, ${ }^{1}$ and A. S. Vasenko ${ }^{1,9,}$ \\ ${ }^{1}$ National Research University Higher School of Economics, 101000 Moscow, Russia \\ ${ }^{2}$ Moscow Institute of Physics and Technology, 141700 Dolgoprudny, Russia \\ ${ }^{3}$ Dukhov Research Institute of Automatics (VNIIA), 127055 Moscow, Russia \\ ${ }^{4}$ Faculty of Science and Technology and MESA+ Institute for Nanotechnology, University of Twente, 7500 AE Enschede, The Netherlands \\ ${ }^{5}$ Donostia International Physics Center (DIPC), Paseo Manuel de Lardizabal 4, San Sebastián/Donostia, 20018 Basque Country, Spain \\ ${ }^{6}$ Departamento de Física de Materiales, Facultad de Ciencias Químicas, UPV/EHU, 20080 San Sebastián, Basque Country, Spain \\ ${ }^{7}$ IKERBASQUE, Basque Foundation for Science, 48011 Bilbao, Spain \\ ${ }^{8}$ N.S. Kurnakov Institute of General and Inorganic Chemistry, Russian Academy of Sciences, 117901 Moscow, Russia \\ ${ }^{9}$ I.E. Tamm Department of Theoretical Physics, P.N. Lebedev Physical Institute, Russian Academy of Sciences, 119991 Moscow, Russia
}

(Received 25 May 2019; revised manuscript received 25 July 2019; published 3 September 2019)

\begin{abstract}
We investigate the behavior of the critical temperature $T_{c}$ in superconductor/ferromagnet/superconductor $(\mathrm{S} / \mathrm{F} / \mathrm{S})$ trilayers in the dirty limit as a function of the ferromagnetic layer thickness $d_{f}$ and the $\mathrm{S} / \mathrm{F}$ interface transparency. We perform $T_{c}$ calculations using the general self-consistent multimode approach based on the Usadel equations in Matsubara Green's functions technique, and compare the results with the singlemode approximation, widely used in literature. Both methods produce similar results for sufficiently low interface transparency. For transparent interfaces, we obtain a qualitatively different $T_{c}\left(d_{f}\right)$ behavior. Using the multimode approach, we observe multiple $0-\pi$ transitions in critical temperature, which cannot be resolved by the single-mode approximation. We also calculate the critical S layer thickness at given $d_{f}$ when an S/F/S trilayer still has a nonzero critical temperature. Finally, we establish the limits of applicability of the single-mode approximation.
\end{abstract}

DOI: 10.1103/PhysRevB.100.104502

\section{INTRODUCTION}

Nowadays, the rates of development in such areas as spintronics, superconducting logic, and memory circuits increase significantly. In particular, much attention is attracted to superconductor/ferromagnet $(\mathrm{S} / \mathrm{F})$ structures [1-3]. It is known that $\mathrm{S} / \mathrm{F}$ structures are important for rapid single flux quantum circuits [4], applications for superconducting spintronics [5], and, in particular, memory elements [6-12] and spin-valves [13-19], magnetoelectronics [20-22], qubits [23], artificial neural networks [24], microrefrigerators [25,26], etc.

Rich physics of $\mathrm{S} / \mathrm{F}$ systems is based on the proximity effect in $\mathrm{S} / \mathrm{F}$ bilayers [1-3,27-30]. It turns out that when a superconductor and a ferromagnet form a hybrid structure, superconducting correlations leak into a ferromagnetic metal over the distance $\xi_{h}=\sqrt{D_{f} / h}$, where $D_{f}$ is the diffusion coefficient and $h$ is the exchange field in the ferromagnetic material [1]. As a consequence, it leads to a damped oscillatory behavior of superconducting correlations in the ferromagnetic layer, with characteristic lengths of decay and oscillations given by $\xi_{h}$.

If a ferromagnetic layer serves as a weak link in a Josephson-type superconductor/ferromagnet/superconductor $(\mathrm{S} / \mathrm{F} / \mathrm{S})$ structure, there is a possibility of a $\pi$ phase state realization. For small $\mathrm{F}$ layer thickness $d_{f} \ll \xi_{h}$, the pair wave function in the $F$ layer is almost constant and the signs of the superconducting pair potentials in the $\mathrm{S}$ layers remain the

*avasenko@hse.ru same. In this case, the phase difference between the $\mathrm{S}$ layers is zero (0 phase state). Increasing the $\mathrm{F}$ layer thickness up to $d_{f} \sim \xi_{h}$, the pair wave function may cross zero in the center of the F layer with the $\pi$ phase shift and different signs of the superconducting pair potential in the $S$ layers $(\pi$ phase state). Further increasing of $d_{f}$ may cause subsequent $0-\pi$ transitions due to damped oscillatory behavior of the pair potential in the F layer. The existence of the $\pi$ states leads to a number of striking phenomena. For example, the critical current in $\mathrm{S} / \mathrm{F} / \mathrm{S}$ Josephson junctions exhibits a damped oscillatory behavior with increasing the $F$ layer thickness [31-49]. The $\pi$ state is then characterized by a negative sign of the critical current. Similarly, 0 to $\pi$ transitions can also be observed as density of states oscillations [50-53] and critical temperature $T_{c}$ oscillations [54-69] in $\mathrm{S} / \mathrm{F} / \mathrm{S}$ trilayers with increasing $d_{f}$. Zero to $\pi$ transitions were also obtained in $\mathrm{F}^{\prime} / \mathrm{S} / \mathrm{F} / \mathrm{S}$ structures with a magnetization misalignment in $\mathrm{F}$ and $\mathrm{F}^{\prime}$ layers $[70,71]$. We also mention that spin-orbit coupling effects can dramatically change spectroscopic signatures of Josephson $\mathrm{S} / \mathrm{F} / \mathrm{S}$ junctions. For example, it was shown that in the presence of intrinsic spin-orbit coupling a giant proximity effect due to spin-triplet Cooper pairs can develop in diffusive $S / F / S$ junctions in the $\pi$ phase state [72]. S/F/S Josephson $\pi$ junctions have been proposed as elements of novel superconducting nanoelectronics in many applications like the aforementioned memory elements and qubits.

The critical temperature also has a nontrivial behavior in S/F bilayers [73-78]. In this case, the transition to the $\pi$ phase is impossible, but the commensurability effect between the period of the superconducting correlations oscillation $\left(\sim \xi_{h}\right)$ and 


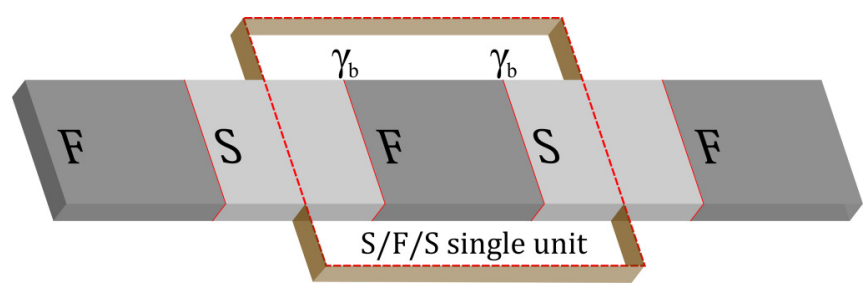

FIG. 1. Geometry of the considered system. A single symmetric $\mathrm{S} / \mathrm{F} / \mathrm{S}$ trilayer is considered in our model. It can also be an elementary unit of the infinite periodic $\mathrm{S} / \mathrm{F}$ multilayer system. The transparency parameter $\gamma_{b}$ is proportional to the resistance across the $\mathrm{S} / \mathrm{F}$ interface.

the $\mathrm{F}$ layer thickness leads to a nonmonotonic $T_{c}\left(d_{f}\right)$ dependence. For the transparent $\mathrm{S} / \mathrm{F}$ interface, $T_{c}$ decays monotonically, vanishing at finite $d_{f}$. With decreasing interface transparency, the critical temperature demonstrates a reentrant behavior: It vanishes in a certain interval of $d_{f}$ and is finite otherwise. At sufficiently low interface transparency, $T_{c}$ decays nonmonotonically to a finite value exhibiting a minimum at a particular $d_{f}$ [73]. Nonmonotonic $T_{c}\left(d_{f}\right)$ dependencies were also observed in $\mathrm{F} / \mathrm{F}^{\prime} / \mathrm{S}$ and $\mathrm{F} / \mathrm{S} / \mathrm{F}^{\prime}$ spin valves with a magnetization misalignment in $\mathrm{F}$ and $\mathrm{F}^{\prime}$ layers [79-81]. Depairing currents in $\mathrm{S} / \mathrm{F}$ proximity structures were studied in Ref. [82].

As already mentioned, in contrast to bilayers, the $S / F / S$ trilayers may exhibit more complex behavior, with the competition of 0 and $\pi$ phase states. The purpose of this paper is to provide a quantitative model of the critical temperature $T_{c}\left(d_{f}\right)$ behavior in a symmetric $\mathrm{S} / \mathrm{F} / \mathrm{S}$ trilayer structure as a function of the ferromagnetic layer thickness $d_{f}$ and the $\mathrm{S} / \mathrm{F}$ interface transparency. Such a structure may be also considered as a single unit of the infinite periodic $\mathrm{S} / \mathrm{F}$ multilayer system as shown in Fig. 1. The total $\mathrm{S} / \mathrm{F}$ multilayer can then be in the 0 or in $\pi$ state, depending on the state of a single $S / F / S$ unit. It should be noted that the $\mathrm{S} / \mathrm{F}$ multilayer system may host states, corresponding to different, more complex configurations of the distribution of superconducting correlations [62-64]. We did not consider these more exotic states in our paper, which could be a subject of future work.

Previously the $T_{c}\left(d_{f}\right)$ behavior of the $\mathrm{S} / \mathrm{F} / \mathrm{S}$ trilayers was studied only in the so-called single-mode approximation (SMA) [54-69]. In this paper, we calculate the $T_{c}\left(d_{f}\right)$ dependence, using the multimode approach (MMA), considered to be an exact method for solving this problem. We also compare the results of the multimode approach with the SMA, setting the limits for the latter approximate method (see the Appendix). In our paper, we do not consider nonequilibrium effects [83], and use the Matsubara Green's functions technique, which has been developed to describe many-body systems in equilibrium at finite temperature [84].

The paper is organized as follows. In Sec. II, we formulate the theoretical model and basic equations. In Secs. III and IV, SMAs and MMAs are formulated, correspondingly. The results are presented and discussed in Sec. V and concluded in Sec. VI.

\section{MODEL}

We consider the $\mathrm{S} / \mathrm{F} / \mathrm{S}$ trilayer depicted in Fig. 2 consisting of a ferromagnetic layer of thickness $d_{f}$ and two superconducting layers of thickness $d_{s}$ along the $x$ direction. The structure is symmetric and its center is placed at $x=0$.

To calculate the critical temperature $T_{c}\left(d_{f}\right)$ of this structure, we assume the diffusive limit and use the framework of the linearized Usadel equations for the $\mathrm{S}$ and $\mathrm{F}$ layers in Matsubara representation [84,85]. Near $T_{c}$, the normal Green's function is $G=\operatorname{sgn} \omega_{n}$, and the Usadel equation for the anomalous Green's function $F$ takes the following form. In the $S$ layers $\left(d_{f} / 2<|x|<d_{s}+d_{f} / 2\right)$ it reads

$$
\xi_{s}^{2} \pi T_{c s} \frac{d^{2} F_{s}}{d x^{2}}-\left|\omega_{n}\right| F_{s}+\Delta=0 .
$$

In the $\mathrm{F}$ layer $\left(-d_{f} / 2<x<d_{f} / 2\right)$, the Usadel equation can be written as

$$
\xi_{f}^{2} \pi T_{c s} \frac{d^{2} F_{f}}{d x^{2}}-\left(\left|\omega_{n}\right|+i h \operatorname{sgn} \omega_{n}\right) F_{f}=0 .
$$

Finally, the self-consistency equation reads [84]

$$
\Delta \ln \frac{T_{c s}}{T}=\pi T \sum_{\omega_{n}}\left(\frac{\Delta}{\left|\omega_{n}\right|}-F_{s}\right) .
$$

In Eqs. (1)-(3), $\xi_{s}=\sqrt{D_{s} / 2 \pi T_{c s}}, \xi_{f}=\sqrt{D_{f} / 2 \pi T_{c s}}, D_{s}$ is the diffusion coefficient in the $\mathrm{S}$ layers, $\omega_{n}=2 \pi T\left(n+\frac{1}{2}\right)$, where $n=0, \pm 1, \pm 2, \ldots$ are the Matsubara frequencies, $h$ is the exchange field in the ferromagnet, $T_{c s}$ is the critical temperature, and $\Delta$ is the pairing potential in the $\mathrm{S}$ layers, and $F_{S(f)}$ denotes
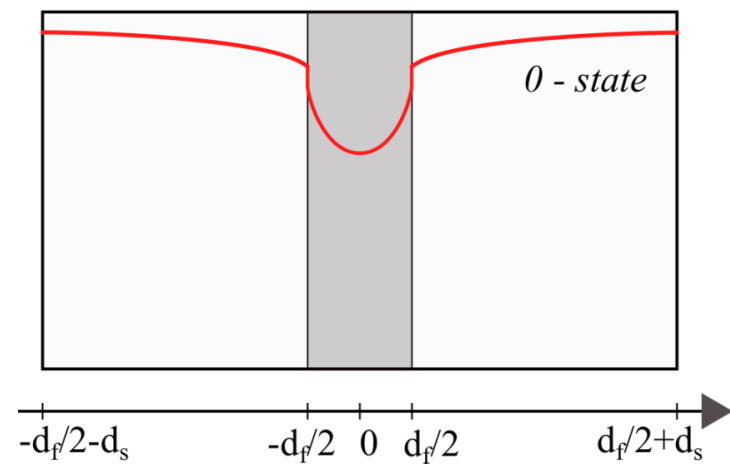

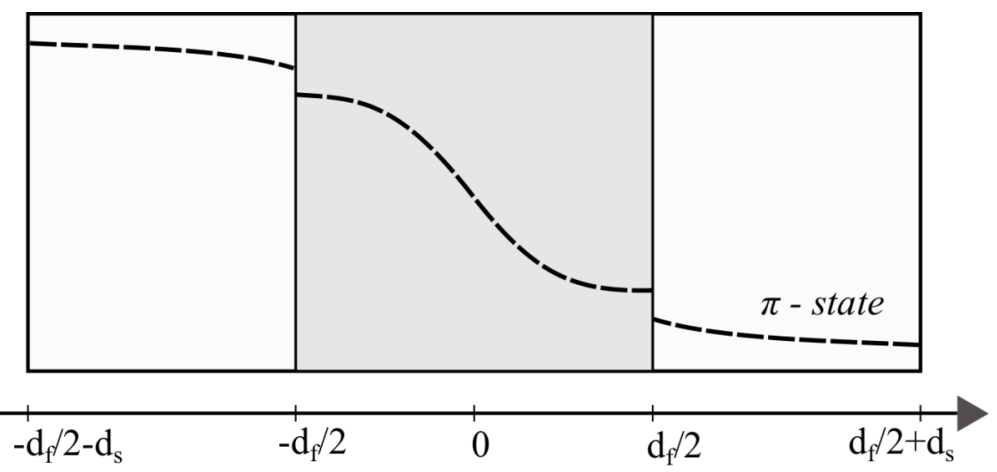

FIG. 2. Schematic behavior of the real part of the pair wave function. For a thin enough ferromagnetic layer, the system is in the 0 phase state (solid red line), while for larger $d_{f}$ the system can be in the $\pi$ state (dashed black line). Only one of these states is realized, depending on the F layer thickness. 
the anomalous Green's function in the $\mathrm{S}(\mathrm{F})$ region (we assume $\left.\hbar=k_{B}=1\right)$. We note that $\xi_{h}=\xi_{f} \sqrt{2 \pi T_{c s} / h}$.

Equations (1)-(3) should be complemented by the Kupriyanov-Lukichev boundary conditions at the $\mathrm{S} / \mathrm{F}$ boundaries $\left(x= \pm d_{f} / 2\right)[86]$ :

$$
\begin{aligned}
\xi_{s} \frac{d F_{s}\left( \pm d_{f} / 2\right)}{d x} & =\gamma \xi_{f} \frac{d F_{f}\left( \pm d_{f} / 2\right)}{d x} \\
\xi_{f} \gamma_{b} \frac{d F_{f}\left( \pm d_{f} / 2\right)}{d x} & = \pm F_{s}\left( \pm d_{f} / 2\right) \mp F_{f}\left( \pm d_{f} / 2\right) .
\end{aligned}
$$

In Eqs. (4), the dimensionless parameter $\gamma=\xi_{s} \sigma_{n} / \xi_{f} \sigma_{s}$ determines the strength of suppression of superconductivity in the $S$ layers near the $S / F$ interfaces compared to the bulk (inverse proximity effect). No suppression occurs for $\gamma=0$, while strong suppression takes place for $\gamma \ll 1$. Here $\sigma_{s(n)}$ is the normal-state conductivity of the $\mathrm{S}(\mathrm{F})$ layer. The dimensionless parameter

$$
\gamma_{b}=R_{b} \sigma_{n} / \xi_{f}
$$

describes the effect of the interface barrier [86,87]. Here $R_{b}$ is the resistance of the $\mathrm{S} / \mathrm{F}$ boundary (we suppose the symmetric structure with same resistance $R_{b}$ for $x= \pm d_{f} / 2$ ).

According to the definition, Eq. (5), $\gamma_{b}=0$ for a fully transparent interface. It follows from Eq. (4b) that the anomalous Green's functions (pair wave functions) $F_{s(f)}$ are continuous at the interface in this case. In the regime of lowbarrier transparency (tunnel junction), $\gamma_{b} \gg 1$ and $F_{s(f)}$ have discontinuities at the interface (see Fig. 2, where finite discontinuity is shown). Lambert et al. have shown that the condition Eq. (4b) is exact in two limits of high and low barrier transparency, $\gamma_{b} \ll 1$ and $\gamma_{b} \gg 1$, correspondingly. They have also found corrections at the intermediate values of $\gamma_{b} \sim 1$ which, however, do not exceed $10 \%$ [88].

At the borders of the $\mathrm{S}$ layers with a vacuum, we naturally have

$$
\frac{d F_{s}\left( \pm d_{s} \pm d_{f} / 2\right)}{d x}=0 .
$$

The solution of the Usadel equation in the F layer depends on the phase state of the structure. In the 0 phase state, the anomalous Green's function is symmetric relative to $x=0$ (see Fig. 2, left panel) [73],

$$
F_{f}^{0}=C\left(\omega_{n}\right) \cosh \left(k_{f} x\right),
$$

while in the $\pi$ phase state the anomalous Green's function is antisymmetric (see Fig. 2, right panel),

$$
F_{f}^{\pi}=C^{\prime}\left(\omega_{n}\right) \sinh \left(k_{f} x\right)
$$

where

$$
k_{f}=\frac{1}{\xi_{f}} \sqrt{\frac{\left|\omega_{n}\right|+i h \operatorname{sgn} \omega_{n}}{\pi T_{c s}}} .
$$

In Eqs. (7) and (8), the $C\left(\omega_{n}\right)$ and $C^{\prime}\left(\omega_{n}\right)$ are proportionality coefficients to be found from the boundary conditions.

To solve the boundary value problem Eqs. (1)-(6), we use the method proposed in Ref. [73]. At the right $S / F$ boundary $\left(x=d_{f} / 2\right)$ from Eqs. (4) we obtain

$$
\xi_{s} \frac{d F_{s}\left(d_{f} / 2\right)}{d x}=\frac{\gamma}{\gamma_{b}+B_{f}\left(\omega_{n}\right)} F_{s}\left(d_{f} / 2\right),
$$

where $B_{f}\left(\omega_{n}\right)$ can acquire one of two different values, depending on phase state. In the 0 phase state [73],

$$
B_{f}^{0}=\left[k_{f} \xi_{f} \tanh \left(k_{f} d_{f} / 2\right)\right]^{-1},
$$

while in $\pi$ phase state from Eq. (8) we obtain

$$
B_{f}^{\pi}=\left[k_{f} \xi_{f} \operatorname{coth}\left(k_{f} d_{f} / 2\right)\right]^{-1} .
$$

A similar boundary condition can be written at $x=-d_{f} / 2$.

The boundary condition Eq. (10) is complex. To rewrite it in a real form, we use the following relation:

$$
F^{ \pm}=F\left(\omega_{n}\right) \pm F\left(-\omega_{n}\right) .
$$

According to the Usadel Eqs. (1)-(3), there is a symmetry relation $F\left(-\omega_{n}\right)=F^{*}\left(\omega_{n}\right)$, which implies that $F^{+}$is a real while $F^{-}$is a purely imaginary function.

Thus we can consider only positive Matsubara frequencies and express the self-consistency Eq. (3) via the symmetric function $F_{s}^{+}$:

$$
\Delta \ln \frac{T_{c s}}{T}=\pi T \sum_{\omega_{n}>0}\left(\frac{2 \Delta}{\omega_{n}}-F_{s}^{+}\right) .
$$

The problem of determining $T_{c}$ can be then formulated in a closed form with respect to $F_{s}^{+}$. Using the boundary condition Eq. (10) we arrive at the effective boundary conditions for $F_{s}^{+}$ at the boundaries of the right $\mathrm{S}$ layer,

$$
\begin{aligned}
\xi_{s} \frac{d F_{s}^{+}\left(d_{f} / 2\right)}{d x} & =W^{0, \pi}\left(\omega_{n}\right) F_{s}^{+}\left(d_{f} / 2\right), \\
\frac{d F_{s}^{+}\left(d_{s}+d_{f} / 2\right)}{d x} & =0,
\end{aligned}
$$

where we used the notations

$$
\begin{aligned}
W^{0, \pi}\left(\omega_{n}\right) & =\gamma \frac{A_{s}\left(\gamma_{b}+\operatorname{Re} B_{f}^{0, \pi}\right)+\gamma}{A_{s}\left|\gamma_{b}+B_{f}^{0, \pi}\right|^{2}+\gamma\left(\gamma_{b}+\operatorname{Re} B_{f}^{0, \pi}\right)}, \\
A_{s} & =k_{s} \xi_{s} \tanh \left(k_{s} d_{s}\right), \quad k_{s}=\frac{1}{\xi_{s}} \sqrt{\frac{\omega_{n}}{\pi T_{c s}}} .
\end{aligned}
$$

Similar boundary conditions can be written at the boundaries of the left $\mathrm{S}$ layer.

The self-consistency Eq. (14) and boundary conditions Eqs. (15), together with the Usadel equation for $F_{s}^{+}$,

$$
\xi_{s}^{2} \pi T_{c s} \frac{d^{2} F_{s}^{+}}{d x^{2}}-\omega_{n} F_{s}^{+}+2 \Delta=0,
$$

will be used for finding the critical temperature of the $S / F / S$ structure both in 0 and $\pi$ phase states. In general, this problem should be solved numerically.

\section{SINGLE-MODE APPROXIMATION}

In this section, we present the SMA method. The solution of the problem Eqs. (14)-(17) can be searched in the form of the following anzatz:

$$
\begin{aligned}
F_{s}^{+}\left(x, \omega_{n}\right) & =f\left(\omega_{n}\right) \cos \left(\Omega \frac{x-d_{s}-d_{f} / 2}{\xi_{s}}\right), \\
\Delta(x) & =\delta \cos \left(\Omega \frac{x-d_{s}-d_{f} / 2}{\xi_{s}}\right)
\end{aligned}
$$


where $\delta$ and $\Omega$ do not depend on $\omega_{n}$. The above solution automatically satisfies boundary condition Eq. (15b) at $x=$ $d_{s}+d_{f} / 2$. Substituting expression Eqs. (18) into the Eq. (17) we obtain

$$
f\left(\omega_{n}\right)=\frac{2 \delta}{\omega_{n}+\Omega^{2} \pi T_{c s}} .
$$

To determine the critical temperature $T_{c}$, we have to substitute Eqs. (18)-(19) into the self-consistency Eq. (14) at $T=T_{c}$. Then it is possible to rewrite the self-consistency Eq. (14) in the following form:

$$
\ln \frac{T_{c s}}{T_{c}}=\psi\left(\frac{1}{2}+\frac{\Omega^{2}}{2} \frac{T_{c s}}{T_{c}}\right)-\psi\left(\frac{1}{2}\right),
$$

where $\psi$ is the digamma function:

$$
\psi(z) \equiv \frac{d}{d z} \ln \Gamma(z), \quad \Gamma(z)=\int_{0}^{\infty} \eta^{z-1} e^{-\eta} d \eta .
$$

Boundary condition Eq. (15a) at $x=d_{f} / 2$ yields the following equation for $\Omega$ :

$$
\Omega \tan \left(\Omega \frac{d_{s}}{\xi_{s}}\right)=W^{0, \pi}\left(\omega_{0}\right),
$$

where we have written $W^{0, \pi}\left(\omega_{0}\right)$ instead of $W^{0, \pi}\left(\omega_{n}\right)$ on the right-hand side, because the left-hand side must be $\omega_{n}$ independent and only zero Matsubara frequency $\omega_{0}$ should be taken into account.

The critical temperature $T_{c}$ is determined by Eqs. (20) and (22) for both 0 and $\pi$ phase states. These equations extend the model of Ref. [73], taking into account the possibility of $\pi$ phase state realization in the considered structure. Although SMA is popular, it is often used without pointing out the limits of its applicability. We derive these limits in the Appendix.

\section{MULTIMODE APPROACH}

The SMA implies that one takes the (only) real root $\Omega$ of Eq. (20). An exact multimode method for solving the problem Eqs. (14)-(17) is obtained if we also take imaginary roots into account (there is infinite number of these, but numerically we take some finite number). The MMA was applied for the first time considering the problem of $T_{c}$ in an $\mathrm{S} / \mathrm{N}$ bilayer [89].

We do not present here the derivation of the MMA. We refer the reader to Ref. [73] [Sec. III, Eqs. (19)-(26) therein] and use similar notations. The solution of the problem Eqs. (15)-(17) within the MMA reduces then to the equation

$$
\operatorname{det} \hat{K}^{0, \pi}=0 \text {, }
$$

where the $\hat{K}$ matrix is defined as

$$
\begin{aligned}
K_{n 0}^{0, \pi} & =\frac{W^{0, \pi}\left(\omega_{n}\right) \cos \left(\Omega_{0} d_{s} / \xi_{s}\right)-\Omega_{0} \sin \left(\Omega_{0} d_{s} / \xi_{s}\right)}{\omega_{n} / \pi T_{c s}+\Omega_{0}^{2}} \\
K_{n m}^{0, \pi} & =\frac{W^{0, \pi}\left(\omega_{n}\right)+\Omega_{m} \tanh \left(\Omega_{m} d_{s} / \xi_{s}\right)}{\omega_{n} / \pi T_{c s}-\Omega_{m}^{2}}
\end{aligned}
$$

where $n=0,1, \ldots, N$ is the index of a Matsubara frequency and $m=1,2, \ldots, M$ is the index of an imaginary root $\Omega_{m}\left[\Omega_{0}\right.$ is the (only) real root]. We take $M=N$. The roots $\Omega_{n}$ are determined by the following equation, obtained from Eq. (14)

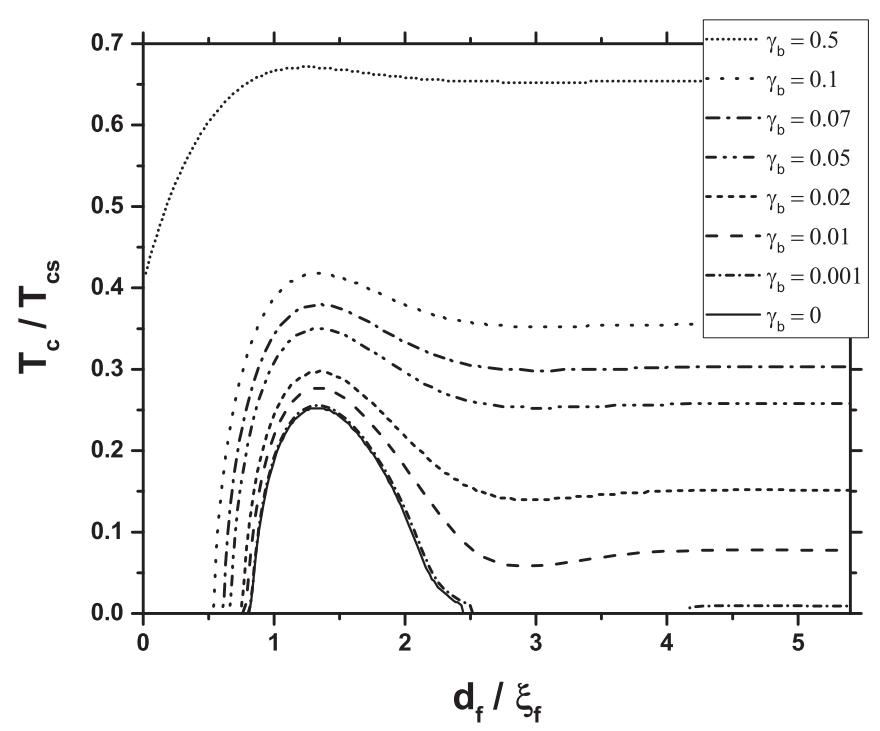

FIG. 3. $T_{c}\left(d_{f}\right)$ dependencies for the $\mathrm{S} / \mathrm{F} / \mathrm{S}$ structure in the $\pi$ phase state, calculated by the multimode approach. $T_{c}$ is normalized by $T_{c s}$, which is the critical temperature of superconductor in the absence of ferromagnetic layer. We also normalize $d_{f}$ by the $\xi_{f}$. Each curve corresponds to particular value of transparency parameter $\gamma_{b}$. Other parameters are mentioned in the text.

at $T=T_{c}$ :

$$
\ln \frac{T_{c s}}{T_{c}}=\psi\left(\frac{1}{2}+\frac{\Omega_{n}^{2}}{2} \frac{T_{c s}}{T_{c}}\right)-\psi\left(\frac{1}{2}\right) .
$$

The MMA is considered to be much more accurate compared to the SMA, and it was shown in previous studies that in some cases, SMA and MMA perform significantly different qualitative behavior for 0 phase state junctions in $\mathrm{S} / \mathrm{F}$ bilayers [73]. In the following, using the MMA, we provide calculations of the critical temperature for various parameters of the $\mathrm{S} / \mathrm{F} / \mathrm{S}$ structure both in 0 and $\pi$ phase states.

\section{RESULTS}

In this section, we present the results obtained by numerical calculations for 0 and $\pi$ phase states using both the SMA and MMA. We provide complete theory for $T_{c}\left(d_{f}\right)$ behavior description in the general case, where systems can be in 0 or $\pi$ phase states, depending on the $\mathrm{F}$ layer thickness $d_{f}$. Moreover, comparison between the SMA and MMA is also presented. The accuracy of calculations was checked by choosing sufficiently large matrix $\hat{K}$ dimensions in MMA. Here and below, we have used in our calculations the same parameters as in Ref. [73], i.e., $\gamma=0.15, h=6.8 \pi T_{c s}, d_{s}=1.24 \xi_{s}$.

\section{A. $T_{c}$ in $\mathrm{S} / \mathrm{F} / \mathrm{S}$ structures in $\pi$ phase state}

In Fig. 3, the critical temperature $T_{c}\left(d_{f}\right)$ dependencies on ferromagnetic layer thickness $d_{f}$ in the $\pi$ phase state calculated by the MMA are shown. This situation corresponds to an $\mathrm{S} / \mathrm{F} / \mathrm{S}$ structure enclosed in a ring, where the $\pi$ phase shift can be fixed by applying the magnetic flux quantum for any $d_{f}$. Different curves correspond to various values of $\gamma_{b}$, 


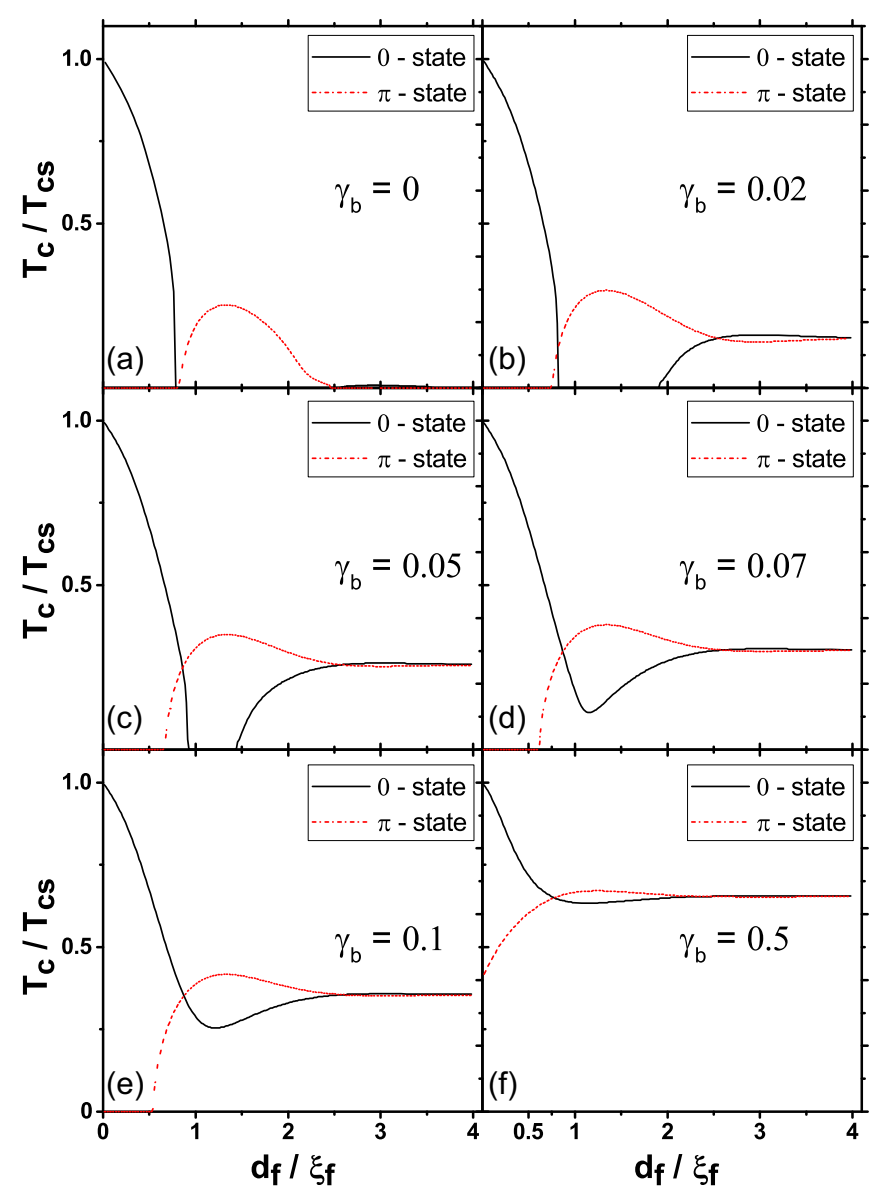

FIG. 4. Plots of $T_{c}\left(d_{f}\right)$ dependencies in both 0 and $\pi$ phase states calculated by the multimode approach. Solid black lines correspond to the 0 phase state, while dashed red lines to the $\pi$ phase state. Each plot corresponds to a particular value of transparency parameter $\gamma_{b}$ : (a) $\gamma_{b}=0$, (b) $\gamma_{b}=0.02$, (c) $\gamma_{b}=0.05$, (d) $\gamma_{b}=0.07$, (e) $\gamma_{b}=0.1$, (f) $\gamma_{b}=0.5$.

which is proportional to resistance across the $\mathrm{S} / \mathrm{F}$ interface [see Eq. (5)] and can be determined from the experiment [78].

For fully transparent $\mathrm{S} / \mathrm{F}$ interfaces, $\gamma_{b}=0$, the critical temperature appears at $d_{f} \sim \xi_{h}$ (we note that in our case $\xi_{h}=0.54 \xi_{f}$, since $h=6.8 \pi T_{c s}$ ), reaches a maximum at a particular $d_{f}$, and with further increase in $d_{f}$ eventually drops to zero. If we consider $\gamma_{b}=0.001$, we may see the reentrant behavior of the $\pi$ phase state, as it first also vanishes but then reappears at larger $d_{f}$ with exponentially dumped amplitude, and finally saturates at small finite value (see also Fig. 5 in logarithmic scale) (we note that at $\gamma_{b}=0$, we do not observe the reentrant behavior due to vanishingly small amplitude of the reentrant $\pi$ phase state). We can explain this behavior as follows. At $\gamma_{b}=0$, all electronic transport through the structure is governed only by the Andreev reflections. In this case, the critical temperature $T_{c}$ vanishes when the $\pi$ phase state becomes energetically unfavorable in a certain interval of $d_{f}$, and at $d_{f} \gg \xi_{f}$ the $T_{c}$ eventually tends to zero. At larger $\gamma_{b}$, the Andreev reflections mix with normal reflections and the inverse proximity effect becomes less pronounced. Therefore, the critical temperature $T_{c}$ at each $d_{f}$ is larger than $T_{c}$ at $\gamma_{b}=$ 0 . Still, at moderately small $\gamma_{b}$, we observe similar behavior:

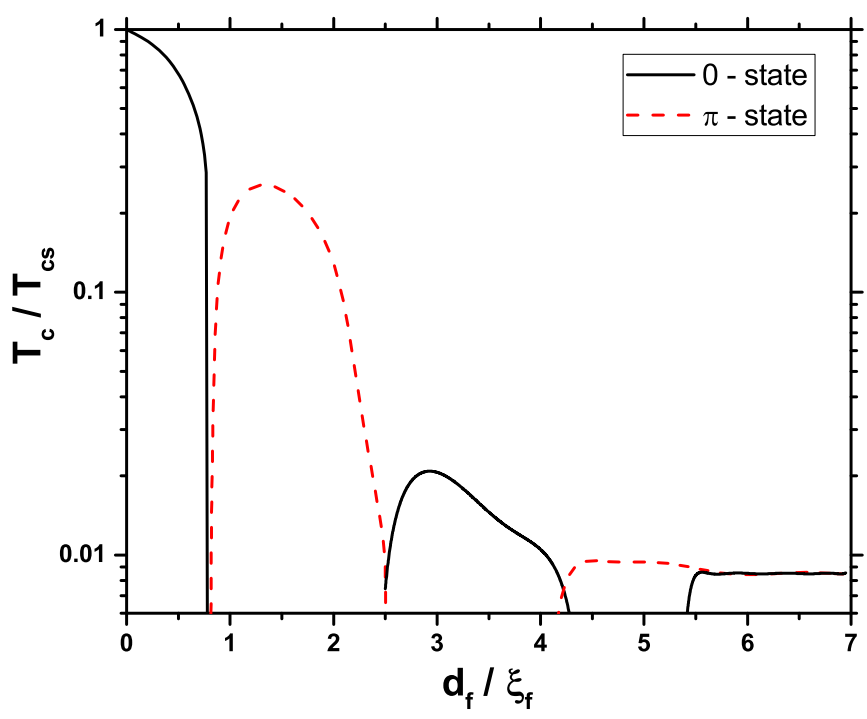

FIG. 5. Illustration of the possibility of multiple $0-\pi$ transitions in case of $\gamma_{b}=0.001$. Calculations are made by the multimode approach.

$T_{c}\left(d_{f}\right)$ reaches a maximum at a particular $d_{f}$ and then decays nonmonotonically and saturates to some value, depending on $\gamma_{b}$ (we note that the oscillatory behavior for large $d_{f}$ can not be seen due to vanishingly small amplitudes of the oscillations). For $\gamma_{b}=0.1 \div 0.2$, one can see the dip on $T_{c}\left(d_{f}\right)$ curve when the $\pi$ phase state is energetically unfavorable. For larger $\gamma_{b}$, this minimum is not resolved due to large contribution of normal reflections at $\mathrm{S} / \mathrm{F}$ interfaces and strong suppression of the inverse proximity effect in $\mathrm{S}$ layers.

\section{B. $T_{c}$ in $\mathrm{S} / \mathrm{F} / \mathrm{S}$ structures: $0-\pi$ transitions}

To provide complete behavior of the critical temperature in $\mathrm{S} / \mathrm{F} / \mathrm{S}$ trilayers, we calculate $T_{c}\left(d_{f}\right)$ dependencies in both 0 and $\pi$ phase states by using the MMA and show them on the same plot, see Fig. 4. Both dependencies are calculated for the same set of parameters mentioned above. In $S / F / S$ trilayers, only the state with highest $T_{c}$ is realized at certain $d_{f}$, i.e., when increasing $d_{f}$ the dashed red line appears above the solid black line, the $0-\pi$ transition occurs, and the structure switches to the $\pi$ phase state. With further increase of $d_{f}$, one can see subsequent $0-\pi$ transitions in the $T_{c}\left(d_{f}\right)$ curve, and in the limit of long $\mathrm{F}$ layer the $T_{c}\left(d_{f}\right)$ saturates at some finite value, depending on $\gamma_{b}$.

The critical temperature $T_{c}\left(d_{f}\right)$ dependencies in the $\pi$ phase state (shown by dashed red lines in Fig. 4) were already discussed above. Let us discuss now the critical temperature behavior in the 0 phase state (shown by solid black lines in Fig. 4). First of all, one can see the reentrant behavior in the 0 phase state only in the case of highly transparent $\mathrm{S} / \mathrm{F}$ interfaces [Figs. 4(a)-4(c)]. At $d_{f} \sim \xi_{h}$, the critical temperature vanishes and then reappear at larger $d_{f}$ with exponentially dumped amplitude. This is similar to the case of $\mathrm{S} / \mathrm{F}$ bilayers [73]. As was mentioned above for small $\gamma_{b}$, the Andreev reflections at $\mathrm{S} / \mathrm{F}$ interfaces are dominant, while for larger $\gamma_{b}$ they mix with normal reflections and the inverse proximity effect is suppressed. Therefore, with increasing $\gamma_{b}$, 


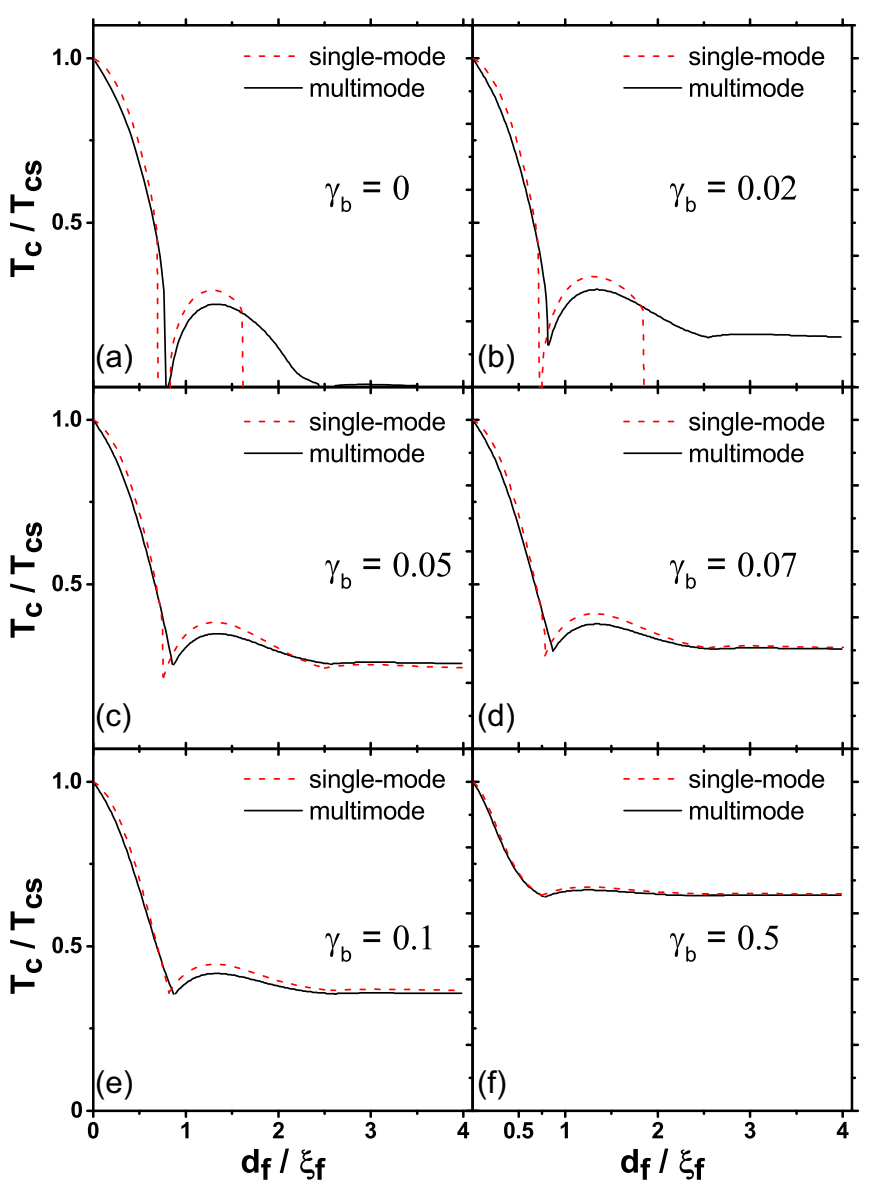

FIG. 6. The comparison between the single-mode approximation and the multimode approach for $\mathrm{S} / \mathrm{F} / \mathrm{S}$ systems. The set of parameters is the same as in Fig. 3. It is clear that for large enough values of $\gamma_{b}$, both single and multimode approaches perform very close results [(d)-(f)], while there are quantitative and even qualitative differences for small $\gamma_{b}[(\mathrm{a}),(\mathrm{b})]$.

the critical temperature in the 0 phase state at each $d_{f}$ is also increasing, similar to $T_{c}$ in the $\pi$ phase state. At moderately low values of $\gamma_{b}$ \{Figs. 4(d) and 4(e)], the $T_{c}\left(d_{f}\right)$ curve in the 0 phase state has a dip in a certain interval of $d_{f}$, when the 0 phase is energetically unfavorable and the $\pi$ phase is realized. This nonmonotonic $T_{c}\left(d_{f}\right)$ curve having a minimum is also similar to the case of S/F bilayers [73]. However, the physical mechanisms are different in these cases. While the reason for the minimum of the $T_{c}\left(d_{f}\right)$ curve in the 0 phase state in $\mathrm{S} / \mathrm{F} / \mathrm{S}$ trilayers is the $0-\pi$ transition, in an $\mathrm{S} / \mathrm{F}$ bilayer this mechanism is irrelevant, and the cause of the minimum is the quasiparticle interference, specific to $S / F$ structures [73].

Multiple 0- $\pi$ transitions upon the increase of the $F$ layer thickness in the case of high S/F interface transparency $\left(\gamma_{b}=\right.$ 0.001 ) are shown in Fig. 5. Further increase of $d_{f}$ leads to the saturation of the critical temperature. In this region, the $0-\pi$ transitions are undistinguishable due to the exponential decay of the anomalous wave function in the ferromagnetic layer [as follows from Eq. (9)].

Summarizing the above results, one can clearly see the difference in $T_{c}\left(d_{f}\right)$ behavior for different values of $\gamma_{b}$ parameter. For low values of $\gamma_{b}$ [high interface transparency], the $T_{c}\left(d_{f}\right)$

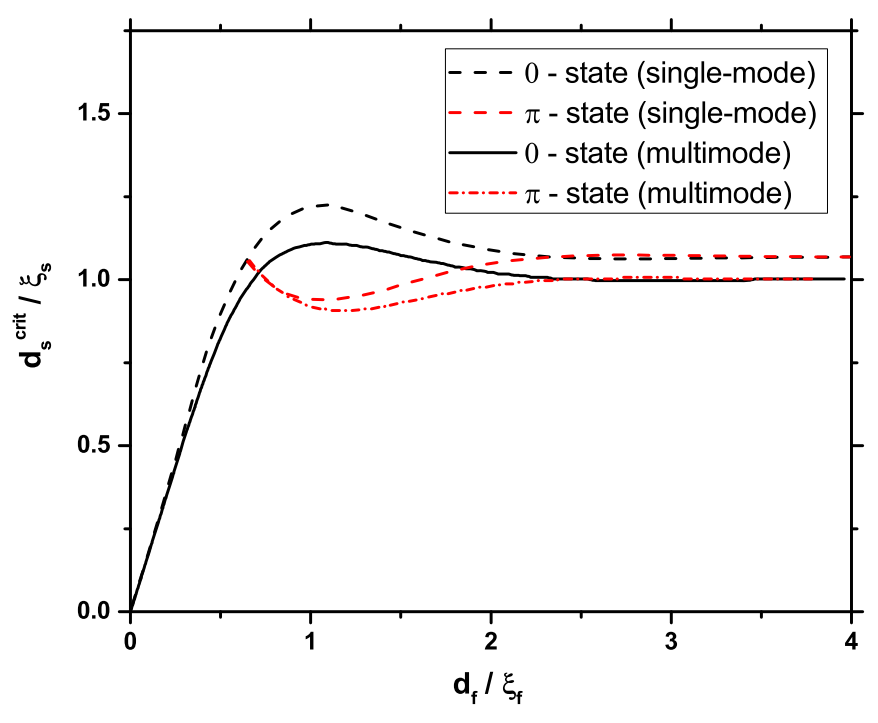

FIG. 7. The $d_{s}^{\text {crit }}\left(d_{f}\right)$ dependence calculated by the single-mode approximation and the multimode approach. The $\gamma_{b}=0.1$, other parameters are same as in Fig. 6.

curve exhibits the highly pronounced dumped oscillations. With increase of $\gamma_{b}$, the amplitude of oscillations decreases and curves are smeared.

\section{Comparison of SMA and MMA}

The SMA is frequently used to calculate the critical temperature. However, this approach has restricted range of applicability which was considered in Ref. [73] for S/F bilayers. Nevertheless, the SMA method is used quite often for a wide range of parameters due to its simplicity and speed, even in the regime when this approximation is not applicable.

The difference between SMA and MMA can be seen in Fig. 6. This figure shows the effective $T_{c}\left(d_{f}\right)$ in an $\mathrm{S} / \mathrm{F} / \mathrm{S}$ trilayer, corresponding to the highest $T_{c}$ which is actually realized at given $d_{f}$, calculated by using both the SMA and the MMA. For these calculations, the same set of parameters as in Fig. 4 was used. It is important to emphasize that, though for large enough values of $\gamma_{b}$, both approximations present close and sometimes almost similar results [Figs. 6(d)6(f)], for small enough interface resistance they are quantitatively [Fig. 6(c)] and even qualitatively different [Figs. 6(a) and 6(b)]. Within the SMA, the $T_{c}\left(d_{f}\right)$ drops to zero abruptly at certain $d_{f}$ in the latter case. In contrast, the MMA provides finite values of $T_{c}$ even at large $d_{f}$ as can be seen from Fig. 6. For small enough values of $d_{f}$, both approximations demonstrate similar results even in case of high interface transparency. From this, we can conclude that the multiple $0-\pi$ transition picture (Fig. 5) cannot be resolved by using the SMA.

The limits of applicability of the SMA are derived for both 0 and $\pi$ phase states in the Appendix.

\section{Critical thickness of the $S$ layer}

With decrease of the S-layer thickness $d_{s}$ in S/F/S trilayers, the critical temperature is suppressed due to the inverse proximity effect, which becomes more profound in the case 
of small $d_{s}$. Hence at certain value $d_{s}$ for a given thickness of ferromagnetic layer $d_{f}$, the critical temperature $T_{c}$ drops to zero, i.e., superconductivity in the structure vanishes. In Fig. 7, the $d_{s}^{\text {crit }}\left(d_{f}\right)$ dependence is shown both for 0 and $\pi$ phase states of the structure, calculated using SMA and MMA. The S/F/S structure chooses the corresponding phase state $(0$ or $\pi)$ to minimize its energy. Such lowest energy state corresponds to a smallest critical value of the S-layer thickness, $d_{s}^{\text {crit }}$. It can be seen from the figure that $d_{s}^{\text {crit }}\left(d_{f}\right)$ also demonstrates the dumped oscillatory behavior.

\section{CONCLUSIONS}

In this paper, we have calculated $T_{c}\left(d_{f}\right)$ dependencies in $\mathrm{S} / \mathrm{F} / \mathrm{S}$ trilayers for different values of transparency parameter $\gamma_{b}$. Comparing the results obtained by both SMA and the MMA, we note that for the low transparent S/F interfaces both methods show good agreement with each other. However, for highly transparent interfaces, SMA and MMA provide quantitatively and even qualitatively different results. In fact, in this case, in SMA, the critical temperature drops to zero abruptly at particular $d_{f}$ [see Figs. 6(a) and 6(b)], while in MMA the superconducting state is still present. This is one of the most important results of our work. Using MMA, we have observed multiple $0-\pi$ transitions in critical temperature, which cannot be resolved by the single-mode approximation (see Fig. 5). We have also calculated the dependence of the critical S-layer thickness on $d_{f}$ (see Fig. 7). This is a minimal thickness of superconducting layers at given $d_{f}$ when an $\mathrm{S} / \mathrm{F} / \mathrm{S}$ trilayer still has a nonzero critical temperature. Finally, we have derived the limits of applicability of the SMA for both 0 and $\pi$ states (see the Appendix). Thus we confirm the importance of using the MMA in a wide range of parameters in the case of $\mathrm{S} / \mathrm{F} / \mathrm{S}$ trilayers, where $0-\pi$ phase transitions are possible.

Finally, we would like to list possible extensions of this work. One of the interesting problems would be to extend the MMA to the nonequilibrium case by using the KeldyshUsadel Green's function approach [84]. It is also important to apply the MMA to calculate the dependence of the critical temperature in $\mathrm{S} / \mathrm{F}$ multilayers on magnetization misalignments in neighboring $\mathrm{F}$ layers. In case of thin enough superconducting layers $\left(\sim \xi_{h}\right)$, the long-range triplet correlations appear in these structures [3] and influence the critical temperature behavior. It is also interesting to study more complex phases in S/F multilayers in the MMA, extending the results of Ref. [63] obtained in the SMA. Other possible extensions will include spin-orbit coupling effects in equilibrium [72] and nonequilibrium cases [90] and considering $T_{c}$ in $\mathrm{S} / \mathrm{F} / \mathrm{S}$ junctions in the presence of an equilibrium supercurrent [91].

\section{ACKNOWLEDGMENTS}

The authors thank Yu. N. Khaydukov and M. Yu. Kupriyanov for useful discussions. T.K. and A.S.V. acknowledges the support of Project No. T3-93 Quantum Solid State Systems, carried out within the framework of the Basic Research Program at the National Research University Higher School of Economics (HSE) in 2018. V.S.S. acknowledges the support of the Russian Science Foundation (Project No. 18-72-10118). A.A.G. acknowledges partial support by the Twinning project SPINTECH under Grant Agreement No. 810144.

\section{APPENDIX: APPLICABILITY OF THE SINGLE-MODE APPROXIMATION}

As mentioned above, SMA is very popular because of its simplicity and speed. However, this approximation is applicable only in a small range of parameters. The solution of the equations in Sec. II are accurate when considered as $\omega_{n}$ independent, which happens in the case when $\gamma_{b} \gg\left|B_{f}\right|$ in Eq. (10). Estimating $\left|B_{f}\right|$, we introduce the real and imaginary parts of $k_{f}$ in Eqs. (11)-(12), $k_{f}=k_{f}^{\prime}+i k_{f}^{\prime \prime}$, and note that $k_{f}^{\prime} \gg k_{f}^{\prime \prime}$. Then from Eqs. (11)-(12), we obtain

$$
\begin{aligned}
&\left|B_{f}^{0}\right| \sim\left[k_{f}^{\prime} \xi_{f} \tanh \left(k_{f}^{\prime} d_{f}\right)\right]^{-1}, \\
&\left|B_{f}^{\pi}\right| \sim\left[k_{f}^{\prime} \xi_{f} \operatorname{coth}\left(k_{f}^{\prime} d_{f}\right)\right]^{-1},
\end{aligned}
$$

and finally write the condition $\gamma_{b} \gg\left|B_{f}\right|$ for 0 phase state,

$$
\frac{1}{\gamma_{b}} \ll \min \left\{\sqrt{\max \left(\frac{T_{c}}{T_{c s}}, \frac{h}{\pi T_{c s}}\right)} ; \frac{d_{f}}{\xi_{f}} \max \left(\frac{T_{c}}{T_{c s}}, \frac{h}{\pi T_{c s}}\right)\right\},
$$

and for $\pi$ phase state,

$$
\frac{1}{\gamma_{b}} \ll \min \left\{\sqrt{\max \left(\frac{T_{c}}{T_{c s}}, \frac{h}{\pi T_{c s}}\right)} ; \frac{\xi_{f}}{d_{f}}\right\},
$$

where the ratio $T_{c} / T_{c s}$ originates from $\omega_{n} / \pi T_{c s}$ with $\omega_{n} \sim$ $\pi T_{c}$ as the characteristic energy scale. Equations (A2)-(A3) provide the conditions of applicability of SMA for both 0 and $\pi$ phase states.
[1] A. I. Buzdin, Rev. Mod. Phys. 77, 935 (2005).

[2] A. A. Golubov, M. Yu. Kupriyanov, and E. Il'ichev, Rev. Mod. Phys. 76, 411 (2004).

[3] F. S. Bergeret, A. F. Volkov, and K. B. Efetov, Rev. Mod. Phys. 77, 1321 (2005).

[4] H. Hilgenkamp, Supercond. Sci. Technol. 21, 034011 (2008).

[5] J. Linder and J. W. A. Robinson, Nat. Phys. 11, 307 (2015).

[6] T. I. Larkin, V. V. Bol'ginov, V. S. Stolyarov, V. V. Ryazanov, I. V. Vernik, S. K. Tolpygo, and O. A. Mukhanov, Appl. Phys. Lett. 100, 222601 (2012).
[7] I. A. Golovchanskiy, V. V. Bol'ginov, V. S. Stolyarov, N. N. Abramov, A. Ben Hamida, O. V. Emelyanova, B. S. Stolyarov, M. Y. Kupriyanov, A. A. Golubov, and V. V. Ryazanov, Phys. Rev. B 94, 214514 (2016).

[8] S. V. Bakurskiy, N. V. Klenov, I. I. Soloviev, M. Yu. Kupriyanov, and A. A. Golubov, Appl. Phys. Lett. 108, 042602 (2016).

[9] I. I. Soloviev, N. V. Klenov, S. V. Bakurskiy, M. Yu. Kupriyanov, A. L. Gudkov, and A. S. Sidorenko, Beilstein J. Nanotechnol. 8, 2689 (2017). 
[10] R. Caruso, D. Massarotti, A. Miano, V. V. Bolginov, A. Ben Hamida, L. N. Karelina, G. Campagnano, I. V. Vernik, F. Tafuri, V. V. Ryazanov, O. A. Mukhanov, and G. P. Pepe, IEEE Trans. Appl. Supercond. 28, 1800606 (2018).

[11] I. A. Golovchanskiy, N. N. Abramov, V. S. Stolyarov, V. V. Ryazanov, A. A. Golubov, and A. V. Ustinov, J. Appl. Phys. 124, 233903 (2018).

[12] S. V. Bakurskiy, N. V. Klenov, I. I. Soloviev, N. G. Pugach, M. Yu. Kupriyanov, and A. A. Golubov, Appl. Phys. Lett. 113, 082602 (2018).

[13] L. R. Tagirov, Phys. Rev. Lett. 83, 2058 (1999).

[14] M. Alidoust, K. Halterman, and O. T. Valls, Phys. Rev. B 92, 014508 (2015).

[15] K. Halterman and M. Alidoust, Phys. Rev. B 94, 064503 (2016).

[16] K. Halterman and M. Alidoust, Supercond. Sci. Technol. 29, 055007 (2016).

[17] A. Srivastava, L. A. B. Olde Olthof, A. Di Bernardo, S. Komori, M. Amado, C. Palomares-Garcia, M. Alidoust, K. Halterman, M. G. Blamire, and J. W. A. Robinson, Phys. Rev. Appl. 8, 044008 (2017).

[18] K. Halterman and M. Alidoust, Phys. Rev. B 98, 134510 (2018).

[19] M. Alidoust and K. Halterman, Phys. Rev. B 97, 064517 (2018).

[20] B. Baek, W. H. Rippard, S. P. Benz, S. E. Russek, and P. D. Dresselhaus, Nat. Commun. 5, 3888 (2014).

[21] E. C. Gingrich, B. M. Niedzielski, J. A. Glick, Y. Wang, D. L. Miller, R. Loloee, W. P. Pratt, Jr., and N. O. Birge, Nat. Phys. 12, 564 (2016)

[22] I. A. Golovchanskiy, N. N. Abramov, V. S. Stolyarov, I. V. Shchetinin, P. S. Dzhumaev, A. S. Averkin, S. N. Kozlov, A. A. Golubov, V. V. Ryazanov, and A. V. Ustinov, J. Appl. Phys. 123, 173904 (2018).

[23] A. K. Feofanov, V. A. Oboznov, V. V. Bol'ginov, J. Lisenfeld, S. Poletto, V. V. Ryazanov, A. N. Rossolenko, M. Khabipov, D. Balashov, A. B. Zorin, P. N. Dmitriev, V. P. Koshelets, and A. V. Ustinov, Nat. Phys. 6, 593 (2010).

[24] I. I. Soloviev, A. E. Schegolev, N. V. Klenov, S. V. Bakurskiy, M. Yu. Kupriyanov, M. V. Tereshonok, A. V. Shadrin, V. S. Stolyarov, and A. A. Golubov, J. Appl. Phys. 124, 152113 (2018).

[25] A. Ozaeta, A. S. Vasenko, F. W. J. Hekking, and F. S. Bergeret, Phys. Rev. B 85, 174518 (2012).

[26] S. Kawabata, A. Ozaeta, A. S. Vasenko, F. W. J. Hekking, and F. S. Bergeret, Appl. Phys. Lett. 103, 032602 (2013).

[27] E. A. Demler, G. B. Arnold, and M. R. Beasley, Phys. Rev. B 55, 15174 (1997).

[28] A. Ozaeta, A. S. Vasenko, F. W. J. Hekking, and F. S. Bergeret, Phys. Rev. B 86, 060509(R) (2012).

[29] F. S. Bergeret and I. V. Tokatly, Phys. Rev. Lett. 110, 117003 (2013).

[30] I. V. Bobkova and A. M. Bobkov, Phys. Rev. B 95, 184518 (2017).

[31] A. I. Buzdin, L. N. Bulaevskii, and S. V. Panyukov, Pis'ma Zh. Eksp. Teor. Phys. 35, 147 (1982) [JETP Lett. 35, 178 (1982)].

[32] A. I. Buzdin and M. Yu. Kupriyanov, Pis'ma Zh. Eksp. Teor. Phys. 53, 308 (1991) [JETP Lett. 53, 321 (1991)].

[33] V. V. Ryazanov, V. A. Oboznov, A. Yu. Rusanov, A. V. Veretennikov, A. A. Golubov, and J. Aarts, Phys. Rev. Lett. 86, 2427 (2001).

[34] Y. Blum, A. Tsukernik, M. Karpovski, and A. Palevski, Phys. Rev. Lett. 89, 187004 (2002).
[35] H. Sellier, C. Baraduc, F. Lefloch, and R. Calemczuk, Phys. Rev. Lett. 92, 257005 (2004).

[36] A. Bauer, J. Bentner, M. Aprili, M. L. Della-Rocca, M. Reinwald, W. Wegscheider, and C. Strunk, Phys. Rev. Lett. 92, 217001 (2004).

[37] C. Bell, R. Loloee, G. Burnell, and M. G. Blamire, Phys. Rev. B 71, 180501(R) (2005).

[38] F. Born, M. Siegel, E. K. Hollmann, H. Braak, A. A. Golubov, D. Yu. Gusakova, and M. Yu. Kupriyanov, Phys. Rev. B 74, 140501(R) (2006).

[39] G. P. Pepe, R. Latempa, L. Parlato, A. Ruotolo, G. Ausanio, G. Peluso, A. Barone, A. A. Golubov, Y. V. Fominov, and M. Yu. Kupriyanov, Phys. Rev. B 73, 054506 (2006).

[40] V. A. Oboznov, V. V. Bol'ginov, A. K. Feofanov, V. V. Ryazanov, and A. I. Buzdin, Phys. Rev. Lett. 96, 197003 (2006).

[41] M. Weides, M. Kemmler, E. Goldobin, D. Koelle, R. Kleiner, H. Kohlsted, and A. Buzdin, Appl. Phys. Lett. 89, 122511 (2006).

[42] A. A. Bannykh, J. Pfeiffer, V. S. Stolyarov, I. E. Batov, V. V. Ryazanov, and M. Weides, Phys. Rev. B 79, 054501 (2009).

[43] A. S. Vasenko, A. A. Golubov, M. Yu. Kupriyanov, and M. Weides, Phys. Rev. B 77, 134507 (2008).

[44] M. Kemmler, M. Weides, M. Weiler, M. Opel, S. T. B. Goennenwein, A. S. Vasenko, A. A. Golubov, H. Kohlstedt, D. Koelle, R. Kleiner, and E. Goldobin, Phys. Rev. B 81, 054522 (2010).

[45] T. E. Baker, A. Richie-Halford, O. E. Icreverzi, and A. Bill, Europhys. Lett. 107, 17001 (2014).

[46] M. Alidoust and K. Halterman, Phys. Rev. B 89, 195111 (2014).

[47] R. Loria, C. Meneghini, K. Torokhtii, L. Tortora, N. Pompeo, C. Cirillo, C. Attanasio, and E. Silva, Phys. Rev. B 92, 184106 (2015).

[48] S. V. Bakurskiy, V. I. Filippov, V. I. Ruzhickiy, N. V. Klenov, I. I. Soloviev, M. Yu. Kupriyanov, and A. A. Golubov, Phys. Rev. B 95, 094522 (2017).

[49] T. Yamashita, A. Kawakami, and H. Terai, Phys. Rev. Appl. 8, 054028 (2017).

[50] T. Kontos, M. Aprili, J. Lesueur, and X. Grison, Phys. Rev. Lett. 86, 304 (2001).

[51] A. Buzdin, Phys. Rev. B 62, 11377 (2000).

[52] K. Halterman and O. T. Valls, Phys. Rev. B 69, 014517 (2004).

[53] A. S. Vasenko, S. Kawabata, A. A. Golubov, M. Yu. Kupriyanov, C. Lacroix, F. S. Bergeret, and F. W. J. Hekking, Phys. Rev. B 84, 024524 (2011).

[54] J. S. Jiang, D. Davidović, D. H. Reich, and C. L. Chien, Phys. Rev. Lett. 74, 314 (1995).

[55] J. S. Jiang, D. Davidović, D. H. Reich, and C. L. Chien, Phys. Rev. B 54, 6119 (1996).

[56] M. G. Khusainov and Y. N. Proshin, Phys. Rev. B 56, R14283(R) (1997).

[57] L. R. Tagirov, Physica C 307, 145 (1998).

[58] M. Khusainov, Y. A. Izyumov and Y. N. Proshin, Physica B 284-288, 503 (2000).

[59] Y. N. Proshin, Y. A. Izyumov, and M. G. Khusainov, Phys. Rev. B 64, 064522 (2001).

[60] Y. A. Izyumov, Y. N. Proshin, and M. G. Khusainov, UFN 172, 113 (2002); Yu. A. Izyumov, Yu. N. Proshin, and M. G. Khusainov, Phys. Usp. 45, 109 (2002). 
[61] C. Cirillo, C. Bell, G. Iannone, S. L. Prischepa, J. Aarts, and C. Attanasio, Phys. Rev. B 80, 094510 (2009).

[62] P. H. Barsic, O. T. Valls, and K. Halterman, Phys. Rev. B 73, 144514 (2006); 75, 104502 (2007).

[63] V. N. Kushnir and M. Yu. Kupriyanov, Pis'ma Zh. Eksp. Teor. Phys. 93, 597 (2011) [JETP Lett. 93, 539 (2011)].

[64] V. N. Kushnir, A. S. Sidorenko, L. R. Tagirov, and M. Yu. Kupriyanov, in Functional Nanostructures and Metamaterials for Superconducting Spintronics, NanoScience and Technology, edited by A. Sidorenko (Springer, Cham, Switzerland, 2018), pp. 1-29; L. R. Tagirov, M. Yu. Kupriyanov, A. S. Sidorenko, and V. N. Kushnir, ibid., pp. 31-47.

[65] V. N. Kushnir, S. L. Prischepa, C. Cirillo, A. Vecchione, C. Attanasio, M. Yu. Kupriyanov, and J. Aarts, Phys. Rev. B 84, 214512 (2011).

[66] A. V. Samokhvalov and A. I. Buzdin, Phys. Rev. B 92, 054511 (2015).

[67] V. N. Kushnir and M. Yu. Kupriyanov, Low Temp. Phys. 42, 900 (2016).

[68] S. N. Vdovichev, Y. N. Nozdrin, E. E. Pestov, P. A. Yunin, and A. V. Samokhvalov, JETP Lett. 104, 329 (2016).

[69] Y. N. Khaydukov, A. S. Vasenko, E. A. Kravtsov, V. V. Progliado, V. D. Zhaketov, A. Csik, Y. V. Nikitenko, A. V. Petrenko, T. Keller, A. A. Golubov, M. Yu. Kupriyanov, V. V. Ustinov, V. L. Aksenov, and B. Keimer, Phys. Rev. B 97, 144511 (2018).

[70] Y. N. Proshin, A. Zimin, N. G. Fazleev, and M. G. Khusainov, Phys. Rev. B 73, 184514 (2006).

[71] M. Avdeev, M. Khusainov, Y. Proshin, and S. Tsarevskii, Supercond. Sci. Technol. 23, 105005 (2010).

[72] S. H. Jacobsen and J. Linder, Phys. Rev. B 92, 024501 (2015).

[73] Y. V. Fominov, N. M. Chtchelkatchev, and A. A. Golubov, Phys. Rev. B 66, 014507 (2002).

[74] C. Cirillo, S. L. Prischepa, M. Salvato, C. Attanasio, M. Hesselberth, and J. Aarts, Phys. Rev. B 72, 144511 (2005).

[75] V. Zdravkov, A. Sidorenko, G. Obermeier, S. Gsell, M. Schreck, C. Müller, S. Horn, R. Tidecks, and L. R. Tagirov, Phys. Rev. Lett. 97, 057004 (2006).
[76] V. I. Zdravkov, J. Kehrle, G. Obermeier, S. Gsell, M. Schreck, C. Müller, H.-A. Krug von Nidda, J. Lindner, J. MoosburgerWill, E. Nold, R. Morari, V. V. Ryazanov, A. S. Sidorenko, S. Horn, R. Tidecks, and L. R. Tagirov, Phys. Rev. B 82, 054517 (2010).

[77] V. I. Zdravkov, J. Kehrle, G. Obermeier, A. Ullrich, S. Gsell, D. Lenk, C. Müller, R. Morari, A. S. Sidorenko, and V. V. Ryazanov, Supercond. Sci. Technol. 24, 095004 (2011).

[78] D. Mancusi, E. A. Ilyina, V. N. Kushnir, S. L. Prischepa, C. Cirillo, and C. Attanasio, J. Appl. Phys. 110, 113904 (2011).

[79] J. Zhu, I. N. Krivorotov, K. Halterman, and O. T. Valls, Phys. Rev. Lett. 105, 207002 (2010).

[80] Y. V. Fominov, A. A. Golubov, T. Yu. Karminskaya, M. Yu. Kupriyanov, R. G. Deminov, and L. R. Tagirov, Pis'ma Zh. Eksp. Teor. Phys. 91, 329 (2010) [JETP Lett. 91, 308 (2010)].

[81] C.-T. Wu, O. T. Valls, and K. Halterman, Phys. Rev. B 86, 014523 (2012).

[82] J. M. E. Geers, M. B. S. Hesselberth, J. Aarts, and A. A. Golubov, Phys Rev B 64, 094506 (2001).

[83] K. Yu. Arutyunov, S. A. Chernyaev, T. Karabassov, D. S. Lvov, V. S. Stolyarov, and A. S. Vasenko, J. Phys.: Condens. Matter 30, 343001 (2018).

[84] W. Belzig, F. K. Wilhelm, C. Bruder, G. Schön, and A. D. Zaikin, Superlattices Microstruct. 25, 1251 (1999).

[85] K. D. Usadel, Phys. Rev. Lett. 25, 507 (1970).

[86] M. Yu. Kuprianov and V. F. Lukichev, Zh. Eksp. Teor. Fiz. 94, 139 (1988) [Sov. Phys. JETP 67, 1163 (1988)].

[87] E. V. Bezuglyi, A. S. Vasenko, V. S. Shumeiko, and G. Wendin, Phys. Rev. B 72, 014501 (2005); E. V. Bezuglyi, A. S. Vasenko, E. N. Bratus, V. S. Shumeiko, and G. Wendin, ibid. 73, 220506(R) (2006).

[88] C. J. Lambert, R. Raimondi, V. Sweeney, and A. F. Volkov, Phys. Rev. B 55, 6015 (1997).

[89] A. A. Golubov, M. Yu. Kupriyanov, V. F. Lukichev, and A. A. Orlikovskii, Mikroelektronika 12, 355 (1983) [Sov. J. Microelectron. 12, 191 (1984).

[90] S. H. Jacobsen and J. Linder, Phys. Rev. B 96, 134513 (2017).

[91] S. H. Jacobsen, I. Kulagina, and J. Linder, Sci. Rep. 6, 23926 (2016). 